TRANSACTIONS OF THE

AMERICAN MATHEMATICAL SOCIETY

Volume 365, Number 10, October 2013, Pages 5525-5544

S 0002-9947(2013)05934-X

Article electronically published on May 10, 2013

\title{
THE MONOTONICITY OF THE RATIO OF TWO ABELIAN INTEGRALS
}

\author{
CHANGJIAN LIU AND DONGMEI XIAO
}

ABstract. In this paper, we study the monotonicity of the ratio of two Abelian integrals

$$
I_{0}(h)=\int_{\Gamma_{h}} y d x \quad \text { and } \quad I_{1}(h)=\int_{\Gamma_{h}} x y d x,
$$

where $\Gamma_{h}$ is a compact component of the level set $\left\{(x, y): y^{2}+\Psi(x)=\right.$ $h, h \in J\}$; here $J$ is an open interval. We first give a new criterion for determining the monotonicity of the ratio of the above two Abelian integrals. Then using this new criterion, we obtain some new Hamiltonian functions $H(x, y)$ so that the ratio of the associated two Abelian integrals is monotone. Especially when $H(x, y)$ has the form $y^{2}+P_{5}(x)$, we obtain the sufficient and necessary conditions that the ratio of two Abelian integrals is monotone, where $P_{5}(x)$ is a polynomial of $x$ with degree five.

\section{INTRODUCTION}

The weakened 16th Hilbert's problem, proposed by Arnold [1] and 2, is to ask for the lower upper bound of the number of isolated zeros of the Abelian integral

$$
I(h)=\oint_{\Gamma_{h}} f(x, y) d x+g(x, y) d y, h \in J,
$$

if $f(x, y), g(x, y)$ and $H(x, y)$ are polynomials whose degrees are known, and $J \subset R$ is a maximal open interval on which a continuous family of ovals $\Gamma_{h}$ exists, where $\Gamma_{h}$ is a compact component of the level set $H(x, y)=h$.

In this paper, we consider the Newtonian mechanical system, that is, the Hamiltonian function $H(x, y)$ has the form $H(x, y)=y^{2}+\Psi(x)$, where $\Psi(x)$ is an analytic function in an open interval. If $\Psi(x)=P_{n+1}(x)$, which is a polynomial of degree $n+1$, and the degrees of $f(x, y)$ and $g(x, y)$ are $m$ which is independent on $n$, then the curves $\Gamma_{h}=\{(x, y): H(x, y)=h\}$ are called elliptic curves as $n \leq 3$, and the associated Abelian integrals $I(h)$ are called elliptic integrals. If $n \geq 4$, then the ovals $\Gamma_{h}$ are called hyperelliptic curves and the associated Abelian integrals $I(h)$ are said to be hyperelliptic integrals.

Received by the editors April 1, 2012.

2010 Mathematics Subject Classification. Primary 34C07, 34C08; Secondary 37G15.

Key words and phrases. Abelian integral, monotonicity, hyperelliptic Hamiltonian.

The first author was partially supported by the NSFC grant (No. 10901117) and pre-research of Soochow University.

The second author was the corresponding author and was partially supported by the NSFC grants (No. 10831003 and No. 10925102) and the Program of Shanghai Subject Chief Scientists (No. 10XD1406200). 
The study of the number of isolated zeros of $I(h)$ has already made many achievements. For over thirty years, such as when $n=2$ for arbitrary $m$, Petrov in [18] showed that $I(h)$ has at most $m-1$ zeros and this upper bound is sharp. When $n=3$, several authors in [19, 20, 21, 27, 16, proved that the number of zeros of $I(h)$ is linear depending on $m$, and Dumortier and $\mathrm{Li}$ in [6, 7, 8, 9, further gave the exact upper bound of the number of zeros of $I(h)$ for $m \leq 3$. In other words, when $n>3, I(h)$ is a hyperelliptic integral and the problem will be much more difficult. In about 1996, Petrov and Khovanskii obtained the following unpublished result: the number of zeros of $I(h)$ is no more than $A(n) m+B(n)$, where $A, B$ are functions depending only on $n$. In [17], Novikov and Yakovenko gave an upper bound $B(n, m)$ of the number of zeros of $I(h)$, but this bound was too excessive, so they did not write it explicitly. Recently, in [4, Binyamini et al. gave the explicit form on this bound, that is, if $m=n-1$, then $I(h)$ has at most $2^{2^{O(n)}}$ isolated zeros. It is clear that this upper bound is not the exact upper bound. Hence, it is an interesting problem whether or not we can get the exact upper bound on hyperelliptic integrals for some small $m$ such as $m=2$.

In [14, Li and Zhang first gave a very nice criterion, which can be used to determine the monotonicity of the ratio of two Abelian integrals, from which one obtained the exact upper bound for the associated Abelian integral as $m=2$. Recently Grau et al. in [11] generalized Li and Zhang's criterion so that it can be used to deal with the Chebyshev property of $m$ Abelian integrals with $m>2$. Their criterion is useful and can work in many independently arising particular cases: elliptic case, hyperelliptic case, and even the non-algebraic case. There have been some results obtained by using this criterion; here we only list [3, 12, 24, for some hyperelliptic Hamiltonian functions of degrees five and six. However, it is very difficult to use this criteria for most Hamiltonian functions with parameters. Hence, for some Hamiltonian functions in 10, this criteria cannot be applied to deal with the number of isolated zeros of hyperelliptic integrals even when $m=2$.

Motivated by these works, in this paper we try to explore the algebraic-geometrical properties of Hamiltonian functions, and develop a new method to solve the number of isolated zeros of some hyperelliptic integrals, which cannot be solved by criteria in [14] and [11. Thanks to some ideas in 15], we obtain a new criterion to guarantee the monotonicity of the ratio of two Abelian integrals. Using the new criterion, we obtain some new Hamiltonian functions $H(x, y)$ so that the ratio of the associated two Abelian integrals is monotone. Especially, when Hamiltonian functions $H(x, y)$ have the form $y^{2}+P_{5}(x)$ in [10, that is, a hyperelliptic Hamiltonian function with $n=4$, and $f(x, y)=\left(\beta_{0}+\beta_{1} x\right) y$ and $g(x, y)=0$, where $\beta_{0}$ and $\beta_{1}$ are any real constants, we obtain the necessary and sufficient condition that the ratio of two Abelian integrals is monotone. Hence, we can list all the cases where the exact upper bound is one for the isolated zeros of the associated hyperelliptic Abelian integral $I(h)$.

This paper is organized as follows. We deduce a new criterion in section 2 on the monotonicity of the ratio of two Abelian integrals. As applications, we first give in section 3 some new Hamiltonian functions $H(x, y)$ so that the ratio of the associated two Abelian integrals is monotone. Then, in the last section, we discuss hyperelliptic Hamiltonian functions of degree five in [10, obtain the necessary and sufficient conditions that the ratio of two Abelian integrals is monotone, and compare our results with some results in [10]. 


\section{THE NEW CRITERION ON MONOTONICITY}

Consider the Hamiltonian function

$$
H(x, y)=y^{2}+\Psi(x),
$$

where $\Psi(x)$ is analytic in some open interval $(\alpha, A)$. Assume that there exists $a \in(\alpha, A)$, such that the following inequality is satisfied

$$
\Psi^{\prime}(x)(x-a)>0 \quad \text { as } \quad x \in(\alpha, A) \backslash\{a\} .
$$

It is easy to verify that, under the above assumption $(\mathrm{H} 1),(0,0)$ is a local minimum.

Let $h_{1}=\Psi(a)$. Then there exists $h_{2}>h_{1}$ such that for any $h \in\left(h_{1}, h_{2}\right)$, the level curve $\Gamma_{h}$ surrounding $(0,0)$ is a compact component of the level set $\{(x, y)$ : $H(x, y)=h\}$.

Without loss of generality, we assume that $\Psi(\alpha)=\Psi(A)=h_{2}$. Then under the above assumption (H1), the maps $\Psi:(\alpha, a) \mapsto\left(h_{1}, h_{2}\right)$ and $\Psi:(a, A) \mapsto\left(h_{1}, h_{2}\right)$ are strictly monotone, respectively, so that they have analytic inverse functions. Denote the associated inverse functions by $\mu(h)$ and $\nu(h)$. In other words,

$$
\Psi(\mu(h)) \equiv \Psi(\nu(h)) \equiv h, h_{1}<h<h_{2},
$$

where $\alpha<\mu(h)<a<\nu(h)<A$; see Figure 1.

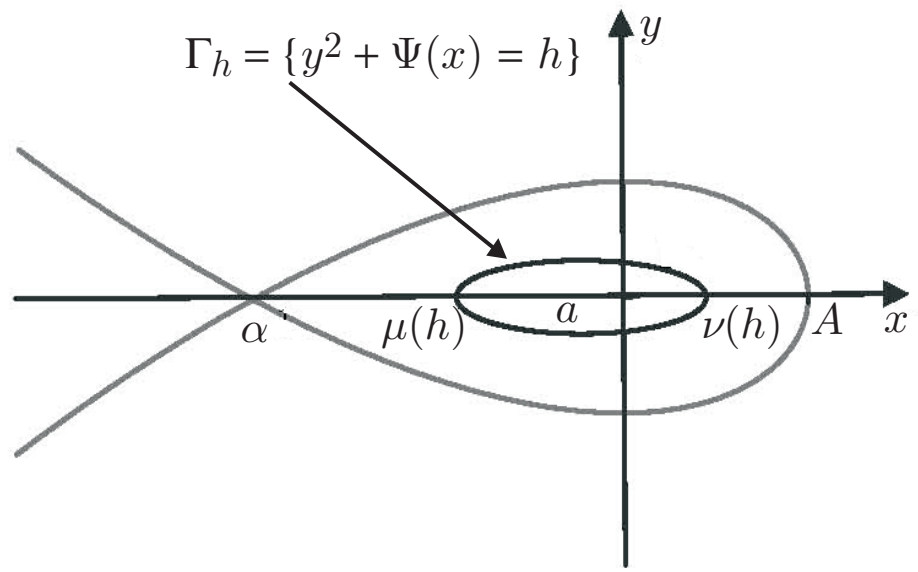

Figure 1. The location of $\mu(h)$ and $\nu(h)$.

Define a function

$$
U(h) \triangleq \mu(h)+\nu(h) .
$$

Then $U(h)$ is an analytic function in $\left(h_{1}, h_{2}\right)$.

Now we consider two Abelian integrals

$$
I_{0}(h)=\oint_{\Gamma_{h}} y d x \quad \text { and } \quad I_{1}(h)=\oint_{\Gamma_{h}} x y d x .
$$

It is easy to check that $I_{0}(h)$ has no zeros in the interval $\left(h_{1}, h_{2}\right)$. Hence, we study the ratio of two Abelian integrals

$$
P(h)=\frac{I_{1}(h)}{I_{0}(h)} .
$$


Our main result is as follows.

Theorem 2.1. Assume that $H(x, y)$ has the form (2.1) and the hypothesis (H1) is satisfied, then $U^{\prime}(h)>0$ (or $\left.U^{\prime}(h)<0\right)$ in $\left(h_{1}, h_{2}\right)$ implies $P^{\prime}(h)>0\left(P^{\prime}(h)<\right.$ 0 , respectively) in $\left(h_{1}, h_{2}\right)$.

Proof. By direct computation, we know that $P^{\prime}(h)$ has the same sign as $I_{1}^{\prime}(h) I_{0}(h)-$ $I_{0}^{\prime}(h) I_{1}(h)$. Hence, we only need to study the sign of $I_{1}^{\prime}(h) I_{0}(h)-I_{0}^{\prime}(h) I_{1}(h)$ in the interval $\left(h_{1}, h_{2}\right)$.

Let

$$
s(h) \triangleq \frac{U(h)}{2}=\frac{\mu(h)+\nu(h)}{2}, r(h) \triangleq \frac{\nu(h)-\mu(h)}{2},
$$

and let $y=y(x) \geq 0$ be a function defined by $y^{2}+\Psi(x)=h$ for $h \in\left(h_{1}, h_{2}\right)$. Then by using the properties of the Hamiltonian function (2.1), we have

$$
\begin{aligned}
& \frac{1}{2}\left(I_{1}^{\prime}(h) I_{0}(h)-I_{0}^{\prime}(h) I_{1}(h)\right) \\
& =\int_{\mu(h)}^{\nu(h)} \frac{x}{y} d x \int_{\mu(h)}^{\nu(h)} y d x-\int_{\mu(h)}^{\nu(h)} \frac{1}{y} d x \int_{\mu(h)}^{\nu(h)} x y d x \\
& =\int_{\mu(h)}^{\nu(h)} \frac{x-s(h)}{y} d x \int_{\mu(h)}^{\nu(h)} y d x-\int_{\mu(h)}^{\nu(h)} \frac{1}{y} d x \int_{\mu(h)}^{\nu(h)}(x-s(h)) y d x .
\end{aligned}
$$

By the change of variable $x=t+s(h)$, we obtain that

$$
\begin{aligned}
\int_{\mu(h)}^{\nu(h)}(x-s(h)) y^{ \pm 1}(x) d x & =\int_{-r(h)}^{r(h)} t y^{ \pm 1}(s(h)+t) d t \\
& =\int_{0}^{r(h)} t\left(y^{ \pm 1}(s(h)+t)-y^{ \pm 1}(s(h)-t)\right) d t \\
\int_{\mu(h)}^{\nu(h)} y^{ \pm 1}(x) d x & =\int_{-r(h)}^{r(h)} y^{ \pm 1}(s(h)+t) d t \\
& =\int_{0}^{r(h)}\left(y^{ \pm 1}(s(h)+t)+y^{ \pm 1}(s(h)-t)\right) d t
\end{aligned}
$$

where $y^{+1}(x)=y$ and $y^{-1}(x)=\frac{1}{y}$.

Since $y^{2}(s(h)+t)+\Psi(s(h)+t)=h$ and $y^{2}(s(h)-t)+\Psi(s(h)-t)=h$,

$$
y^{2}(s(h)+t)-y^{2}(s(h)-t)=\Psi(s(h)-t)-\Psi(s(h)+t) .
$$

We claim that $\Psi(s(h)-t)-\Psi(s(h)+t) \neq 0$ for $t \in(0, r(h))$.

In fact, if there exists a $\bar{t} \in(0, r(h))$ such that $\Psi(s(h)-\bar{t})-\Psi(s(h)+\bar{t})=0$, then $\Psi(s(h)-\bar{t})=\Psi(s(h)+\bar{t})$.

Let $\bar{h}=\Psi(s(h)-\bar{t})$. Then $\mu(\bar{h})=s(h)-\bar{t}$ and $\nu(\bar{h})=s(h)+\bar{t}$. Thus $U(\bar{h})=$ $2 s(h)=U(h)$; this is impossible. Hence, for all $t \in(0, r(h))$, we have

$$
\Psi(s(h)-t)-\Psi(s(h)+t) \neq 0 .
$$

Now we are in a position to prove that $P^{\prime}(h)>0$ if $U^{\prime}(h)>0$.

Suppose that $U^{\prime}(h)>0$ for $h_{1}<h<h_{2}$. Then $U(h)>U\left(h_{1}\right)$, that is, $s(h)>$ $s\left(h_{1}\right)=a$. Let $t_{0}=s(h)-a \in(0, r(h))$. Then $s(h)+t_{0}=2 s(h)-a$ and, by the hypothesis (H1), we know that $\Psi(x)$ is a monotone increasing function in the interval $(a, 2 s(h)-a)$. Thus,

$$
\Psi\left(s(h)-t_{0}\right)-\Psi\left(s(h)+t_{0}\right)=\Psi(a)-\Psi(2 s(h)-a)<0 .
$$


Since $\Psi(s(h)-t)-\Psi(s(h)+t) \neq 0$ for all $t \in(0, r(h)), \Psi(s(h)-t)-\Psi(s(h)+t)$ has the same sign with $\Psi\left(s(h)-t_{0}\right)-\Psi\left(s(h)+t_{0}\right)$, which implies that

$$
\Psi(s(h)-t)-\Psi(s(h)+t)<0 .
$$

Notice that $y^{2}(s(h)+t)+\Psi(s(h)+t)=y^{2}(s(h)-t)+\Psi(s(h)-t)=h$. By (2.5) and the positive of $y(x)$, we have $y(s(h)+t)<y(s(h)-t)$. Therefore, we obtain that

$$
\begin{aligned}
\int_{\mu(h)}^{\nu(h)} \frac{x-s(h)}{y(x)} d x & =\int_{0}^{r(h)} \frac{t(y(s(h)-t)-y(s(h)+t))}{y(s(h)+t) y(s(h)-t)} d t>0, \\
\int_{\mu(h)}^{\nu(h)}(x-s(h)) y(x) d x & =\int_{0}^{r(h)} t(y(s(h)+t)-y(s(h)-t)) d t<0, \\
\int_{\mu(h)}^{\nu(h)} \frac{1}{y(x)} d x & =\int_{0}^{r(h)} \frac{y(s(h)-t)+y(s(h)+t)}{y(s(h)+t) y(s(h)-t)} d t>0, \\
\int_{\mu(h)}^{\nu(h)} y(x) d x & =\int_{0}^{r(h)} \frac{y(s(h)-t)+y(s(h)+t)}{y(s(h)+t) y(s(h)-t)} d t>0 .
\end{aligned}
$$

Hence, $\frac{1}{2}\left(I_{1}^{\prime}(h) I_{0}(h)-I_{0}^{\prime}(h) I_{1}(h)\right)>0$ by (2.4). This leads to

$$
P^{\prime}(h)=\frac{I_{1}^{\prime}(h) I_{0}(h)-I_{0}^{\prime}(h) I_{1}(h)}{I_{0}^{2}(h)}>0 .
$$

We complete the proof that $P^{\prime}(h)>0$ if $U^{\prime}(h)>0$.

Similar arguments can be applied to prove that $P^{\prime}(h)<0$ if $U^{\prime}(h)<0$ for $h_{1}<h<h_{2}$. We omit it here in order to save space.

Remark 2.1. The criterion in [14] is used to determine the monotonicity of the function

$$
\frac{\mu(h) \Psi^{\prime}(\nu(h))-\nu(h) \Psi^{\prime}(\mu(h))}{\Psi^{\prime}(\nu(h))-\Psi^{\prime}(\mu(h))} .
$$

Our criterion is used to determine the monotonicity of the following function

$$
\mu(h)+\nu(h) .
$$

Comparing the two criteria, we can see that our criterion looks simpler when under the same conditions on Hamiltonian functions.

It is clear that the inverse functions $\mu(h)$ and $\nu(h)$ of $\Psi(x)=h$ in the two corresponding intervals cannot usually be written in explicit expressions, which leads to the criterion not being easily applied at times. Next we further assume that the function $\Psi(x)$ for $x \in(\alpha, A)$ has the following asymptotic relation

$$
\Psi^{\prime}(x) \sim(x-a)^{2 k-1}, \quad \text { as } x \rightarrow a,
$$

where $k$ is a natural number. Then we can obtain a direct criterion as follows.

Corollary 2.1. Assume that $H(x, y)$ has the form (2.1) and the hypotheses (H1)(H2) are satisfied. Then $U^{\prime}(h) \neq 0$ for $h \in\left(h_{1}, h_{2}\right)$ if $\xi(x)>0$ for all $x \in$ $(\alpha, A) \backslash\{a\}$ or $\xi(x)<0$ for all $x \in(\alpha, A) \backslash\{a\}$, where

$$
\xi(x)=(x-a)\left((2 k-1) \Psi^{\prime \frac{2 k-2}{2 k-1}}(x)-\Psi^{\prime \prime}(x)\right) .
$$

Therefore, $P(h)$ is monotone if $\xi(x)>0$ (or $\xi(x)<0$ ) for all $x \in(\alpha, A) \backslash\{a\}$. 
Proof. Define a new function

$$
F(x) \triangleq 2 k \Psi(x)-\Psi^{\frac{2 k}{2 k-1}}(x) .
$$

By direct calculation, we have

$$
F^{\prime}(x)=\frac{2 k}{2 k-1} \Psi^{\prime \frac{1}{2 k-1}}(x)\left((2 k-1) \Psi^{\prime \frac{2 k-2}{2 k-1}}(x)-\Psi^{\prime \prime}(x)\right) .
$$

By the hypothesis $(\mathrm{H} 1), \Psi^{\prime}(x)$ has the same sign with $(x-a)$. Thus, $F^{\prime}(x)$ has the same sign with $(x-a)\left((2 k-1) \Psi^{\prime \frac{2 k-2}{2 k-1}}(x)-\Psi^{\prime \prime}(x)\right)$, which is $\xi(x)$. Without loss of generality, we assume that $\xi(x)>0$ for $x \in(\alpha, A) \backslash\{a\}$. Next, we verify $U^{\prime}(h) \neq 0$ for $h \in\left(h_{1}, h_{2}\right)$.

When $\xi(x)>0$ for $x \in(\alpha, A) \backslash\{a\}$, we have that $F^{\prime}(x)>0$ as $x \in(\alpha, A) \backslash\{a\}$. Since $\nu(h)>a>\mu(h)$ for $h \in\left(h_{1}, h_{2}\right)$, we obtain that $F(\nu(h))>F(\mu(h))$, that is,

$$
2 k \Psi(\nu(h))-\Psi^{\frac{2 k}{2 k-1}}(\nu(h))>2 k \Psi(\mu(h))-\Psi^{\prime \frac{2 k}{2 k-1}}(\mu(h)) .
$$

Note that $\Psi(\mu(h))=\Psi(\nu(h))=h$. Hence, $\Psi^{\frac{2 k}{2 k-1}}(\nu(h))<\Psi^{\frac{2 k}{2 k-1}}(\mu(h))$. This implies that

$$
\left|\Psi^{\prime}(\mu(h))\right| \neq\left|\Psi^{\prime}(\nu(h))\right| .
$$

On the other hand, by the hypothesis (H1), we know that $\Psi^{\prime}(\mu(h)) \Psi^{\prime}(\nu(h)) \neq 0$. Since $\Psi(\mu(h))=\Psi(\nu(h))=h$, we obtain that

$$
\mu^{\prime}(h)=\frac{1}{\Psi^{\prime}(\mu(h))}, \nu^{\prime}(h)=\frac{1}{\Psi^{\prime}(\nu(h))} .
$$

Note that $U^{\prime}(h)=\mu^{\prime}(h)+\nu^{\prime}(h)$ for $h \in\left(h_{1}, h_{2}\right)$. Hence, by (2.7)

$$
U^{\prime}(h)=\frac{1}{\Psi^{\prime}(\mu(h))}+\frac{1}{\Psi^{\prime}(\nu(h))} \neq 0
$$

for $h \in\left(h_{1}, h_{2}\right)$.

Moreover, we have that either $U^{\prime}(h)>0$ or $U^{\prime}(h)<0$ if $U^{\prime}(h) \neq 0$ as $h \in\left(h_{1}, h_{2}\right)$. From Theorem 2.1, we know that $P(h)$ is monotone in the interval $\left(h_{1}, h_{2}\right)$. We finish the proof.

\section{Applications of Corollary 2.1}

As applications of our new direct criterion, we give three kinds of Hamiltonian functions, which could be polynomials or non-polynomials, so that the ratio of the associated two Abelian integrals is monotone in this section.

The first Hamiltonian function is

$$
H_{1}(x, y)=y^{2}+\Psi_{1}(x), \quad \Psi_{1}^{\prime}(x)=x\left(1+a_{1} x\right)^{n_{1}}\left(1+a_{2} x\right)^{n_{2}} \cdots\left(1+a_{p} x\right)^{n_{p}},
$$

where $0<a_{p}<a_{p-1}<\cdots<a_{1}, n_{1}, n_{2}, \cdots, n_{p}$ are non-negative real numbers and $p$ is a natural number. We have the following conclusion.

Proposition 3.1. $P_{1}(h)=\frac{\oint_{\Gamma_{1}(h)} x y d x}{\oint_{\Gamma_{1}(h)} y d x}$ is monotone in the interval $\left(0, h_{1}\right)$, where $\Gamma_{1}(h)$ is a compact component of the level set $\left\{(x, y): H_{1}(x, y)=h, h \in\left(0, h_{1}\right)\right\}$ and $h_{1}=\Psi_{1}\left(-\frac{1}{a_{1}}\right)>0$. 
Proof. We consider the Hamiltonian function $H_{1}(x, y)$, and we let $A$ be the $x$ coordinate of the cross point between the compact component $\Gamma_{1}\left(h_{1}\right)$ and the positive $x$-axis.

It is easy to check that the hypotheses (H1) and (H2) in Corollary 2.1 are satisfied if we set $a=0, \alpha=-\frac{1}{a_{1}}$ and $k=1$. Thus, $\xi(x)$ defined in (2.6) has the following form

$$
\xi(x)=x\left(1-\left(1+a_{1} x\right)^{n_{1}}\left(1+a_{2} x\right)^{n_{2}} \cdots\left(1+a_{p} x\right)^{n_{p}}-\sum_{i=1}^{p} n_{i} a_{i} \frac{\Psi_{1}^{\prime}(x)}{1+a_{i} x}\right) .
$$

If $x>0$, then for any $i$ with $1 \leq i \leq p$, we have

$$
1+a_{i} x>1,\left(1+a_{1} x\right)^{n_{1}}\left(1+a_{2} x\right)^{n_{2}} \cdots\left(1+a_{k} x\right)^{n_{k}}>1,
$$

and $n_{i} a_{i} \frac{\Psi_{1}^{\prime}(x)}{1+a_{i} x}$ has the same sign as $\Psi_{1}^{\prime}(x)$, which is positive. So $\xi(x)<0$ for all $x>0$.

On the other hand, if $-\frac{1}{a_{1}}<x<0$, then for any $i$ with $1 \leq i \leq p$, we have

$$
0<1+a_{i} x<1,0<\left(1+a_{1} x\right)^{n_{1}}\left(1+a_{2} x\right)^{n_{2}} \cdots\left(1+a_{k} x\right)^{n_{k}}<1,
$$

and $n_{i} a_{i} \frac{\Psi_{1}^{\prime}(x)}{1+a_{i} x}$ has the same sign as $\Psi_{1}^{\prime}(x)$, which is negative. This implies that $\xi(x)<0$ for all $x$ with $-\frac{1}{a_{1}}<x<0$.

Therefore, $\xi(x)<0$ for all $0 \neq x \in\left(-\frac{1}{a_{1}},+\infty\right)$. By Corollary 2.1, we obtain that $P(h)$ is monotone in the interval $\left(0, h_{1}\right)$. The proof is complete.

The second Hamiltonian function is

$$
\begin{gathered}
H_{2}(x, y)=y^{2}+\Psi_{2}(x), \\
\Psi_{2}^{\prime}(x)=x\left(1+a_{1} x\right)^{n_{1}}\left(1+c_{1} x+b_{1} x^{2}\right)^{m_{1}} \cdots\left(1+c_{q} x+b_{q} x^{2}\right)^{m_{q}},
\end{gathered}
$$

where all parameters $a_{1}, b_{i}, c_{i}$ and $m_{i}$ are positive real numbers, $q$ is a natural number and they all satisfy the following two conditions:

(1) $c_{i}^{2}<4 b_{i}$ for $1 \leq i \leq q$.

(2) $c_{i}>2 a_{1} b_{i}$ for $1 \leq i \leq q$.

In other words, except for zero, all the real parts of the zeros of $\Psi_{2}^{\prime}(x)$ are no more than $-\frac{1}{a_{1}}$. Then we obtain the following conclusion.

Proposition 3.2. $P_{2}(h)=\frac{\oint_{\Gamma_{2}(h)} x y d x}{\oint_{\Gamma_{2}(h)} y d x}$ is monotone in the interval $\left(0, h_{2}\right)$, where $\Gamma_{2}(h)$ is a compact component of the level set $\left\{(x, y): H_{2}(x, y)=h, h \in\left(0, h_{2}\right)\right\}$ and $h_{2}=\Psi_{2}\left(-\frac{1}{a_{1}}\right)>0$.

Proof. It is easy to see that if we set $a=0, \alpha=-\frac{1}{a_{1}}$ and $k=1$, then the hypotheses (H1) and (H2) are satisfied and $(\alpha, A) \subset\left(-\frac{1}{a_{1}},+\infty\right)$. Now $\xi(x)$ defined in (2.6) has the following form

$$
\begin{aligned}
\xi(x)= & x\left(1-\left(1+a_{1} x\right)^{n_{1}}\left(1+c_{1} x+b_{1} x^{2}\right)^{m_{1}} \cdots\left(1+c_{q} x+b_{q} x^{2}\right)^{m_{q}}\right. \\
& \left.-n_{1} a_{1} \frac{\Psi_{2}^{\prime}(x)}{1+a_{1} x}-\sum_{i=1}^{q} m_{i} a_{i} \frac{\Psi_{2}^{\prime}(x)\left(c_{i}+2 b_{i} x\right)}{1+c_{i} x+b_{i} x^{2}}\right) .
\end{aligned}
$$

If $x>0$, then $1+a_{1} x>1$, and for any $i$ with $1 \leq i \leq q$, we have that $1+c_{i} x+b_{i} x^{2}>1,\left(1+a_{1} x\right)^{n_{1}}\left(1+c_{1} x+b_{1} x^{2}\right)^{m_{1}} \cdots\left(1+c_{q} x+b_{q} x^{2}\right)^{m_{q}}>1$, 
and $n_{1} a_{1} \frac{\Psi_{2}^{\prime}(x)}{1+a_{1} x}$ and $m_{i} a_{i} \frac{\Psi_{2}^{\prime}(x)\left(c_{i}+2 b_{i} x\right)}{1+c_{i} x+b_{i} x^{2}}$ have the same sign as $\Psi_{2}^{\prime}(x)$, which is positive. So $\xi(x)<0$ for $x>0$.

On the other hand, if $-\frac{1}{a_{1}}<x<0$, then for any $i$ with $1 \leq i \leq q$, we have that

$$
0<1+a_{i} x<1,0<1+c_{i} x+b_{i} x^{2}<1
$$

and

$$
0<\left(1+a_{1} x\right)^{n_{1}}\left(1+c_{1} x+b_{1} x^{2}\right)^{m_{1}} \cdots\left(1+c_{q} x+b_{q} x^{2}\right)^{m_{q}}<1
$$

by conditions $c_{i}^{2}<4 b_{i}$ and $c_{i}>2 a_{1} b_{i}$ for $1 \leq i \leq q$.

Note that either $n_{1} a_{1} \frac{\Psi_{2}^{\prime}(x)}{1+a_{1} x}$ or $m_{i} a_{i} \frac{\Psi_{2}^{\prime}(x)\left(c_{i}+2 b_{i} x\right)}{1+c_{i} x+b_{i} x^{2}}$ have the same sign as $\Psi_{2}^{\prime}(x)$, which is negative. Thus, $\xi(x)<0$ for $-\frac{1}{a_{1}}<x<0$.

Summing up the above analysis, we obtain that $\xi(x)<0$ for $0 \neq x \in\left(-\frac{1}{a_{1}},+\infty\right)$. By Corollary 2.1, $P(h)$ is monotone in the interval $\left(0, h_{2}\right)$. The proof is complete.

It is clear that both of the Hamiltonian systems associated to the above Hamiltonian functions $H_{1}(x, y)$ and $H_{2}(x, y)$, respectively, have an elementary center at $(0,0)$. Proposition 3.1 (or Proposition 3.2) show that there exists a continuous family $\Gamma_{1}(h)\left(\Gamma_{2}(h)\right.$, respectively) of ovals surrounding the elementary center $(0,0)$ such that the ratio of the corresponding two Abelian integrals is monotone.

In the following, we give the last Hamiltonian function, the associated Hamiltonian system has a nilpotent center at $(0,0)$. The Hamiltonian function is

$$
H_{3}(x, y)=y^{2}+\Psi_{3}(x), \quad \Psi_{3}^{\prime}(x)=x^{2 N-1}(1+x)^{\beta},
$$

where $N>2$ is a natural number, and $\beta \neq 0$ is a real number. Then the following conclusion is true.

Proposition 3.3. $P_{3}(h)=\frac{\oint_{\Gamma_{3}(h)} x y d x}{\oint_{\Gamma_{3}(h)} y d x}$ is monotone in the interval $\left(0, h_{3}\right)$, where $\Gamma_{3}(h)$ is a compact component of the level set $\left\{(x, y): H_{3}(x, y)=h, h \in\left(0, h_{3}\right)\right\}$ and $h_{3}=\Psi_{3}(-1)>0$.

Proof. It is easy to see that if we set $a=0, \alpha=-1$ and $k=N$, then the hypotheses (H1) and (H2) are satisfied and $(\alpha, A) \subset(-1,+\infty)$. By direct calculation, we can see that $\xi(x)$ defined in (2.6) has the following form

$$
\xi(x)=x^{2 N-1}(1+x)^{\beta-1}\left((2 N-1)(1+x)^{\frac{2 N-1-\beta}{2 N-1}}-(2 N-1)(1+x)-\beta x\right) .
$$

Let

$$
g(x)=(2 N-1)(1+x)^{\frac{2 N-1-\beta}{2 N-1}}-(2 N-1)(1+x)-\beta x .
$$

Obviously $g^{\prime}(x)$ has at most one zero in $(-1,+\infty)$, so $g(x)$ has at most two zeros in $(-1,+\infty)$.

If $\beta>0$, then $g(x) \sim-(2 N-1+\beta) x$ when $x \rightarrow+\infty$. So $g(x)<0$ for sufficiently large $x$. On the other hand, when $x \rightarrow-1^{+}, g(x) \geq-(2 N-1)(1+x)-\beta x \rightarrow \beta$. Thus, $g(x)>0$ for $0<x+1 \ll 1$.

Summing up the above analysis, we find that as $\beta>0, g(x)$ has the opposite signs at the two endpoints of the interval $(-1,+\infty)$. Hence, the number of zeros of $g(x)$ must be odd in the interval $(-1,+\infty)$. But $g(x)$ has at most two zeros in $(-1,+\infty)$. This implies that $g(x)$ has exactly one zero in $(-1,+\infty)$.

Notice that $g(0)=0$. Consequently, $x=0$ is the unique zero of $g(x)$ in $(-1,+\infty)$. So, $g(x)>0$ for $x \in(-1,0)$ and $g(x)<0$ for $x \in(0,+\infty)$. 
Whenever $x \in(-1,0)$ or $x \in(0,+\infty)$, we have that $\xi(x)=x^{2 N-1}(1+x)^{\beta-1} g(x)$ $<0$. By Corollary 2.1, $P(h)$ is monotone in $\left(0, h_{3}\right)$.

We omit the proof of the case $\beta<0$ since it is similar. And the proof is complete.

\section{Application of Theorem 2.1}

To our knowledge, the first work on the number of zeros of hyperelliptic integrals of the first kind was done by Gavrilov and Iliev in [10]. There have been some later papers on this topic for some classes of hyperelliptic integrals; see [3, 12, 24, 23, 25. 26. For more recent works, we recommend the paper [13. As an application of Theorem 2.1, we re-consider the Hamiltonian function in 10] and obtain the necessary and sufficient conditions that the ratio of two Abelian integrals associated with this Hamiltonian function is monotone. Consequently we list all the cases that the exact upper bound is one for the isolated zeros of the associated Abelian integral $I(h)=\beta_{0} I_{0}(h)+\beta_{1} I_{1}(h)$.

Consider the Hamiltonian function,

$$
H_{0}(x, y)=y^{2}+\Psi_{0}(x)
$$

where $\Psi_{0}(x)$ is a polynomial of degree five. If the hypothesis (H1) is satisfied in Theorem 2.1] as shown in [10, we can assume, without any loss of generality, that $H_{0}(x, y)$ is in the form

$$
H_{0}(x, y)=y^{2}+\Psi_{0}(x), \Psi_{0}(x)=-\frac{u v}{2} x^{2}+\frac{u+v+u v}{3} x^{3}-\frac{1+u+v}{4} x^{4}+\frac{1}{5} x^{5}
$$

where either $0 \leq u \leq v \leq 1$ or $u=\bar{v} \in \mathbb{C} \backslash \mathbb{R}$.

The associated Hamiltonian system is

$$
\begin{aligned}
& \dot{x}=-2 y, \\
& \dot{y}=x(x-u)(x-v)(x-1) .
\end{aligned}
$$

Obviously, if $u \neq 0, v \neq 1$, then $p_{0}=(0,0)$ is a saddle of system (4.9), and $p_{1}=(1,0)$ is the center of system (4.9). In addition, if $0<u<v<1$, then $p_{v}=(v, 0)$ is a saddle of system (4.9), and $p_{u}=(u, 0)$ is the center of system (4.9). We denote the critical values of $H_{0}(x, y)$ at $p_{0}, p_{u}, p_{v}$ and $p_{1}$ by $h_{0}, h_{u}, h_{v}$ and $h_{1}$, respectively. Hence, we have

$$
\begin{aligned}
& h_{0}=H_{0}(0,0)=0, \\
& h_{1}=H_{0}(1,0)=-\frac{1}{60}(3-5 u-5 v+10 u v), \\
& h_{u}=H_{0}(u, 0)=-\frac{u^{3}}{60}\left(3 u^{2}-5 u v-5 u+10 v\right), \\
& h_{v}=H_{0}(v, 0)=-\frac{v^{3}}{60}\left(3 v^{2}-5 u v-5 v+10 u\right) .
\end{aligned}
$$

If $0 \leq u \leq v \leq 1$, then $h_{0} \geq h_{u}, h_{v} \geq h_{u}, h_{v} \geq h_{1}$. In this case, the possible phase portraits of system (4.9) are shown in the Figure 2. In this figure, we can see that there are three bifurcation lines

$$
\begin{aligned}
& L_{v}=\{(u, v): u=0,0 \leq v \leq 1\}, \\
& L_{u v}=\{(u, v): 0 \leq u=v \leq 1\}, \\
& L_{1}=\{(u, v): v=1,0 \leq u \leq 1\},
\end{aligned}
$$


and a bifurcation curve $\gamma: h_{v}=h_{0}$, that is,

$$
\gamma=\left\{(u, v): u=\frac{3 v^{2}-5 v}{5(v-2)}, 0 \leq v \leq 1\right\}
$$

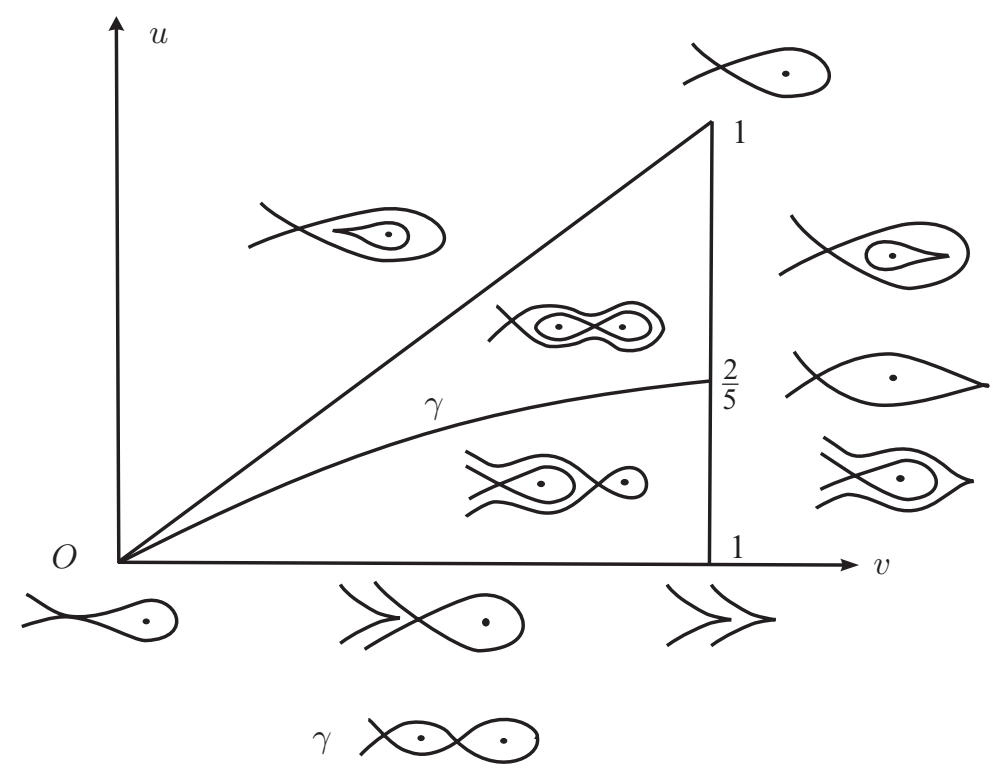

FiguRE 2. Bifurcation diagram of system (4.9) in the real case.

Since it is very difficult to deal with the number of isolated zeros of the Abelian integral associated with $H_{0}(x, y)$ for the general polynomial functions $f(x, y)$ and $g(x, y)$, we only consider the simplest case $f(x, y)=\left(\beta_{0}+\beta_{1} x\right) y$ and $g(x, y)=0$, where $\beta_{0}$ and $\beta_{1}$ are any real constants. In other words, we consider the number of isolated zeros of the following Abelian integral

$$
I(h)=\beta_{0} I_{0}(h)+\beta_{1} I_{1}(h),
$$

where $I_{0}(h)=\oint_{\Gamma_{0}(h)} y d x, I_{1}(h)=\oint_{\Gamma_{0}(h)} x y d x$ and $\Gamma_{0}(h)$ is a compact component of the level set $\left\{(x, y): H_{0}(x, y)=h\right\}$.

It is easy to check that $I_{0}(h)$ has no zeros for all real values of $h$ except some critical values. Thus, we can consider the function $P_{0}(h)=\frac{I_{1}(h)}{I_{0}(h)}$ in an open interval of $h$.

In the following, we shall study the monotonicity of $P_{0}(h)$ when $\Gamma_{0}(h)$ is a continuous family of ovals surrounding only one center, $(1,0)$ or $(u, 0)$ of system (4.9).

We first consider the monotonicity of $P_{0}(h)$ when $\Gamma_{0}(h)$ is a continuous family of ovals surrounding only the center $(1,0)$. Our results are as follows.

Theorem 4.1. Suppose that $\Gamma_{0}(h)$ is a continuous family of ovals surrounding the center $(1,0)$. Then

(i) the function $P_{0}(h)$ is monotone in $\left(h_{1}, h_{v}\right)$ if $0 \leq u \leq v<1$;

(ii) the function $P_{0}(h)$ is monotone in $\left(h_{1}, h_{0}\right)$ if $u=\bar{v}$ and $(\operatorname{Re}(u)-2)^{2}+$ $(\operatorname{Im} v)^{2} \geq 1$; 
(iii) the function $P_{0}(h)$ is not monotone in $\left(h_{1}, h_{0}\right)$ if $u=\bar{v}$ and $(\operatorname{Re}(u)-2)^{2}+$ $(\operatorname{Im} v)^{2}<1$.

Proof. Using the notation $\mu(h), \nu(h), s(h), r(h), U(h)$ in section 2, we will show that $U(h)$ is monotone in $\left(h_{1}, h_{v}\right)$ for case $(\mathrm{i})$, and $U(h)$ is monotone in $\left(h_{1}, h_{0}\right)$ for case (ii).

Note that the compact component $\Gamma_{0}(h)$ of $y^{2}+\Psi_{0}(x)=h$ intersects $y=0$ at the points $(\mu(h), 0)$ and $(\nu(h), 0)$, where $\mu(h)<1<\nu(h)$.

From $\Psi_{0}(\mu(h))=\Psi_{0}(\nu(h))$, it follows that

$$
\int_{1}^{\mu(h)} x(x-u)(x-v)(x-1) d x=\int_{1}^{\nu(h)} x(x-u)(x-v)(x-1) d x,
$$

and

$\int_{0}^{1-\mu(h)} x(1-x-u)(1-x-v)(1-x) d x=\int_{0}^{\nu(h)-1}(1+x)(1+x-u)(1+x-v) x d x$.

If $0 \leq u \leq v<1$, that is, the case (i), then one has $\nu(h)-1<1-\mu(h)$ which leads to $s(h)<1$.

If $u=\bar{v}$ and $(\operatorname{Re} u-2)^{2}+(\operatorname{Im} u)^{2} \geq 1$, that is, the case (ii), then we have

$$
\begin{gathered}
(1+x)(1+x-u)(1+x-v)-(1-x-u)(1-x-v)(1-x) \\
=2 x\left(x^{2}+3-4 \operatorname{Re} u+(\operatorname{Re} u)^{2}+(\operatorname{Im} u)^{2}\right) \geq 0 .
\end{gathered}
$$

Hence we also have $\nu(h)-1<1-\mu(h)$ and $s(h)<1$.

For any $h \in\left(h_{1}, h_{v}\right)$ in case (i) or $h \in\left(h_{1}, h_{0}\right)$ in case (ii), we define a new function

$$
\kappa(t) \triangleq \Psi_{0}(s(h)+t)-\Psi_{0}(s(h)-t), \quad t \in(0, r(h)) .
$$

Obviously $\kappa(t)$ is a polynomial of degree 5 with respective to $t$, and 0 is a root of $\kappa(t)$. Furthermore, $t= \pm r(h)$ are two roots of $\kappa(t)$ since $\mu(h)$ and $\nu(h)$ are roots of $\Psi_{0}(x)=h$. After calculation, we obtain

$$
\kappa(t)=-t\left(\frac{2}{5} t^{4}+\left(\frac{2}{3}(u+v+u v)-2(u+v+1) s(h)+4 s(h)^{2}\right) t^{2}+C\right),
$$

where $C=s(h)(s(h)-u)(s(h)-v)(s(h)-1)<0$.

From (4.10), we can see that the other two roots of $\kappa(t)$ are of the form $\pm t_{1}(h)$ and their product is positive. Therefore, $t_{1}(h) \in \mathbb{C} \backslash \mathbb{R}$ and $t_{1}(h)^{2}<0$.

Summing up the above analysis, we obtain

$$
\kappa(t)=-\frac{2}{5} t(t-r(h))(t+r(h))\left(t^{2}-t_{1}(h)^{2}\right)>0
$$

for all $t \in(0, r(h))$.

Now we claim that $U(h)$ is monotone for $h \in\left(h_{1}, h_{v}\right)$ in case (i) or for $h \in\left(h_{1}, h_{0}\right)$ in case (ii).

By contradiction, we assume that there exists $h^{*}$ and $h^{* *}, h_{1}<h^{*}<h^{* *}<h_{v}$ in case (i) and $h_{1}<h^{*}<h^{* *}<h_{0}$ in case (ii) such that $U\left(h^{*}\right)=U\left(h^{* *}\right)$, which implies that $s\left(h^{*}\right)=s\left(h^{* *}\right)$. It is clear that

$$
0<r\left(h^{*}\right)=\frac{\nu\left(h^{*}\right)-\mu\left(h^{*}\right)}{2}<\frac{\nu\left(h^{* *}\right)-\mu\left(h^{* *}\right)}{2}=r\left(h^{* *}\right) .
$$


Let $h=h^{* *}$ in $\kappa(t)$. Then

$$
\begin{aligned}
\kappa(t) & =\Psi_{0}\left(s\left(h^{* *}\right)+t\right)-\Psi_{0}\left(s\left(h^{* *}\right)-t\right) \\
& =-\frac{2}{5} t\left(t-r\left(h^{* *}\right)\right)\left(t+r\left(h^{* *}\right)\right)\left(t^{2}-t_{1}\left(h^{* *}\right)^{2}\right) .
\end{aligned}
$$

Obviously, $\kappa(t)>0$ for all $t \in\left(0, r\left(h^{* *}\right)\right)$.

Now we calculate $\kappa(t)$ in (4.11) at $t=r\left(h^{*}\right)$ since $0<r\left(h^{*}\right)<r\left(h^{* *}\right)$. By the definition of $\kappa(t)$, we know that

$$
\begin{aligned}
\kappa\left(r\left(h^{*}\right)\right) & =\Psi_{0}\left(s\left(h^{* *}\right)+r\left(h^{*}\right)\right)-\Psi_{0}\left(s\left(h^{* *}\right)-r\left(h^{*}\right)\right) \\
& =\Psi_{0}\left(s\left(h^{*}\right)+r\left(h^{*}\right)\right)-\Psi_{0}\left(s\left(h^{*}\right)-r\left(h^{*}\right)\right) \\
& =\Psi_{0}\left(\nu\left(h^{*}\right)\right)-\Psi_{0}\left(\mu\left(h^{*}\right)\right)=h^{*}-h^{*}=0 .
\end{aligned}
$$

This contradicts $\kappa(t)>0$ for all $t \in\left(0, r\left(h^{* *}\right)\right)$. Therefore, $U(h)$ is monotone for $h \in\left(h_{1}, h_{v}\right)$ in case (i) or $h \in\left(h_{1}, h_{0}\right)$ in case (ii).

By Theorem 2.1, we obtain that $P_{0}(h)$ is monotone in $h \in\left(h_{1}, h_{v}\right)$ if $0 \leq u \leq$ $v<1$, and $P_{0}(h)$ is monotone in $\left(h_{1}, h_{0}\right)$ if $u=\bar{v}$ and $(\operatorname{Re}(u)-2)^{2}+(\operatorname{Im} v)^{2} \geq \overline{1}$. Thus, the conclusions (i) and (ii) are true.

In the following we will show that $P_{0}(h)$ is not monotone in $\left(h_{1}, h_{0}\right)$ if $u=\bar{v}$ and $(\operatorname{Re}(u)-2)^{2}+(\operatorname{Im} v)^{2}<1$.

We first compute the asymptotic expansion of $I_{0}(h)$ and $I_{1}(h)$ at $h=h_{1}$. Here $h_{1}=-\frac{1}{60}(3-5 u-5 v+10 u v)$; that is, we solve the expressions of $I_{0}(h)$ and $I_{1}(h)$ near the center $p_{1}=(1,0)$.

Moving the center $p_{1}$ to the origin, let $X=x-1$, and $Y=y$. We can write system (4.9) in the following form

$$
\begin{aligned}
& \dot{X}=-2 Y, \\
& \dot{Y}=X(X+1-u)(X+1-v)(X+1),
\end{aligned}
$$

where $u=\bar{v}$ and $(\operatorname{Re}(u)-2)^{2}+(\operatorname{Im} v)^{2}<1$.

System (4.12) has only two equilibria: a center at the origin $(0,0)$ and a saddle at $(-1,0)$. The Hamiltonian function is

$H_{01}(X, Y)=Y^{2}+\frac{(1-u)(1-v)}{2} X^{2}+\frac{3-2(u+v)+u v}{3} X^{3}+\frac{3-(u+v)}{4} X^{4}+\frac{1}{5} X^{5}$.

Then $h_{10}=H_{01}(0,0)=0, h_{11}=H_{01}(-1,0)=\frac{1}{120}+\frac{1}{6}\left(\left(\operatorname{Re}(u)-\frac{1}{2}\right)^{2}+(\operatorname{Im} v)^{2}\right)>0$, and the ovals $\gamma_{1}(h)$ are the compact components of $H_{01}(X, Y)=h$ with $h_{10}<h<$ $h_{11}$. Thus, the ovals $\Gamma_{0}(h)$ of $H_{0}(x, y)=h$ with $h_{1}<h<h_{0}$ can be mapped to $\gamma_{1}(h)$ of $H_{01}(X, Y)=h$ with $h_{10}<h<h_{11}$ one-by-one, in which $\Gamma_{0}\left(h_{1}\right)$ corresponds to $\gamma_{1}\left(h_{10}\right)$, a center of system (4.12). For simplicity of notation, we use the same $h$ to represent the corresponding values in the intervals $\left(h_{1}, h_{0}\right)$ and $\left(h_{10}, h_{11}\right)$, respectively,

$$
\begin{aligned}
& I_{0}(h)=\oint_{\Gamma_{0}(h)} y d x=\oint_{\gamma_{1}(h)} Y d X \triangleq I_{10}(h), \\
& I_{1}(h)=\oint_{\Gamma_{0}(h)} x y d x=\oint_{\gamma_{1}(h)}(X+1) Y d X \triangleq I_{11}(h)+I_{10}(h) .
\end{aligned}
$$

Thus, $P_{0}(h)=\frac{I_{1}(h)}{I_{0}(h)}=1+\frac{I_{11}(h)}{I_{10}(h)}$.

Let $P_{10}(h) \triangleq \frac{I_{11}(h)}{I_{10}(h)}$. Then $P_{0}^{\prime}(h)=P_{10}^{\prime}(h)$ in the corresponding interval of $h$. 
Now we calculate $I_{10}(h)$ and $I_{11}(h)$ as $0<h \ll 1$. We first make a transformation of variables in a small neighborhood of $(0,0)$,

$x=X\left(\frac{(1-u)(1-v)}{2}+\frac{3-2(u+v)+u v}{3} X+\frac{3-(u+v)}{4} X^{2}+\frac{1}{5} X^{3}\right)^{\frac{1}{2}}, y=Y$.

Then we have the inverse transformation as follows

$$
X=\phi(x)=\frac{\sqrt{2}}{\sqrt{(1-u)(1-v)}} x-\frac{2(3+u v-2(u+v))}{3(1-u)^{2}(1-v)^{2}} x^{2}+O\left(x^{3}\right), \quad Y=y,
$$

where $O\left(x^{3}\right)$ is the higher order term and the ovals $\gamma_{0}(h)$ have the expression $x^{2}+y^{2}=h$ with $0<h \ll 1$ in the new variables $(x, y)$. Therefore, we can obtain that

$$
\begin{aligned}
I_{10}(h) & =\oint_{\gamma_{1}(h)} Y d X=h \int_{0}^{2 \pi}-\sin ^{2} \theta \phi^{\prime}(\sqrt{h} \cos \theta) d \theta \\
& =-\frac{\sqrt{2} \pi}{\sqrt{(1-u)(1-v)}} h+O\left(h^{2}\right) \\
I_{11}(h) & =\oint_{\gamma_{1}(h)} X Y d X=\int_{0}^{2 \pi}-h \sin ^{2} \theta \phi(\sqrt{h} \cos \theta) \phi^{\prime}(\sqrt{h} \cos \theta) d \theta \\
& =\frac{\sqrt{2} \pi(3+u v-2(u+v))}{2(1-u)^{2}(1-v)^{2} \sqrt{(1-u)(1-v)}} h^{2}+O\left(h^{3}\right),
\end{aligned}
$$

where $\phi^{\prime}(x)=\frac{d \phi(x)}{d x}$, and $O\left(h^{2}\right)$ and $O\left(h^{3}\right)$ are the higher order terms of $h^{2}$ and $h^{3}$, respectively. Hence, we have that

$$
\begin{aligned}
P_{10}^{\prime}(h) & =\frac{I_{11}^{\prime}(h) I_{10}(h)-I_{10}^{\prime}(h) I_{11}(h)}{I_{10}^{2}(h)} \\
& =-\frac{3+u v-2(u+v)}{2(1-u)^{2}(1-v)^{2}}+O(h) .
\end{aligned}
$$

Note that

$$
3+u v-2(u+v)=(\operatorname{Re}(u)-2)^{2}+(\operatorname{Im} v)^{2}-1 .
$$

By the condition $(\operatorname{Re}(u)-2)^{2}+(\operatorname{Im} v)^{2}<1$, we obtain that

$$
P_{0}^{\prime}\left(h_{1}\right)=P_{10}^{\prime}\left(h_{10}\right)=\lim _{h \rightarrow 0^{+}} P_{10}^{\prime}(h)=-\frac{3+u v-2(u+v)}{2(1-u)^{2}(1-v)^{2}}>0 .
$$

On the other hand, we will show that $P_{0}^{\prime}\left(h_{0}\right)<0$. We consider the asymptotic expansion of $I_{0}(h)$ and $I_{1}(h)$ at $h=h_{0}=0$.

For the sake of convenience, we let $X=-x, Y=-y$. Then system (4.9) can be written to

$$
\begin{aligned}
& \dot{X}=-2 Y, \\
& \dot{Y}=-X(X+u)(X+v)(X+1),
\end{aligned}
$$

where $u=\bar{v}$ and $(\operatorname{Re}(u)-2)^{2}+(\operatorname{Im} v)^{2}<1$.

System (4.13) has only two equilibria: a saddle at $(0,0)$ and a center at $(-1,0)$. The Hamiltonian function is

$$
H_{02}(X, Y)=-Y^{2}+\frac{u v}{2} X^{2}+\frac{u+v+u v}{3} X^{3}+\frac{u+v+1}{4} X^{4}+\frac{1}{5} X^{5} .
$$


Then $h_{20}=H_{02}(0,0)=0, h_{21}=H_{02}(-1,0)=\frac{1}{120}+\frac{1}{6}\left(\left(\operatorname{Re}(u)-\frac{1}{2}\right)^{2}+(\operatorname{Im} v)^{2}\right)>0$, and the ovals $\gamma_{2}(h)$ are the compact components of $H_{02}(X, Y)=h$ with $0=h_{20}<$ $h<h_{21}$. Hence, $\Gamma_{0}(h)$ of $H_{0}(x, y)=h$ with $h_{1}<h<h_{0}$ can be mapped to $\gamma_{2}(h)$ of $H_{02}(X, Y)=h$ with $h_{20}<h<h_{21}$ one-by-one, in which $\Gamma_{0}\left(h_{0}\right)$ corresponds to $\gamma_{2}\left(h_{20}\right)$, a homoclinic loop of system (4.13). We have similar arguments in the center case,

$$
\begin{aligned}
& I_{0}(h)=\oint_{\Gamma_{0}(h)} y d x=\oint_{\gamma_{2}(h)} Y d X \triangleq I_{20}(h), \\
& I_{1}(h)=\oint_{\Gamma_{0}(h)} x y d x=\oint_{\gamma_{2}(h)}-X Y d X \triangleq-I_{21}(h) .
\end{aligned}
$$

Following the method in 22, we can obtain the asymptotic expansions of $I_{20}(h)$ and $I_{21}(h)$ as $0<h \ll 1$ as follows,

$$
\begin{aligned}
& I_{20}(h)=a_{20}(h)+b_{20}(h) \ln h=a_{20}(0)+b_{20}^{\prime}(0) h \ln h+O(h), \\
& I_{21}(h)=a_{21}(h)+b_{21}(h) \ln h=a_{21}(0)+a_{21}^{\prime}(0) h+O\left(h^{2} \ln h\right),
\end{aligned}
$$

where $a_{20}(0)=-\oint_{\gamma_{2}(0)} Y d X, b_{20}^{\prime}(0)=-\frac{1}{\sqrt{2 u v}}, a_{21}(0)=-\oint_{\gamma_{2}(0)} X Y d X$ and $a_{21}^{\prime}(0)=-\oint_{\gamma_{2}(0)} \frac{X}{2 Y} d X$.

Thus, we have

$$
P_{0}^{\prime}\left(h_{0}\right)=\lim _{h \rightarrow 0^{+}} \frac{d}{d h}\left(-\frac{I_{21}(h)}{I_{20}(h)}\right)=\lim _{h \rightarrow 0^{+}} \frac{a_{21}(0) b_{20}^{\prime}(0) \ln h}{I_{20}(h)^{2}}=-\infty<0
$$

since $a_{21}(0)<0$ by the Green formula.

Therefore, $P_{0}^{\prime}(h)$ changes sign in the interval $\left(h_{1}, h_{0}\right)$, which leads to that $P_{0}(h)$ is not monotone in the interval $\left(h_{1}, h_{0}\right)$. The proof is complete.

In the following, we study the monotonicity of $P_{0}(h)$ if the continuous family of ovals $\Gamma_{h}$ is surrounding only the center $(u, 0)$ of system (4.9). The conclusions are as follows.

Theorem 4.2. Suppose that $p_{u}=(u, 0)$ is the center of system (4.9) and $P_{0}(h)=$ $\frac{\oint_{\Gamma_{h}} x y d x}{\oint_{\Gamma_{h}} y d x}$, where $\Gamma_{h}$ are ovals surrounding only the center $(u, 0)$. Then $0<u<v \leq 1$ and

(1) $P_{0}(h)$ is monotone in $\left(h_{u}, h_{0}\right)$ if $0<u \leq \frac{1}{3}\left(v+1-\sqrt{v^{2}+v+1}\right)$,

(2) $P_{0}(h)$ is not monotone in $\left(h_{u}, h_{0}\right)$ if $\frac{1}{3}\left(v+1-\sqrt{v^{2}+v+1}\right)<u<\frac{3 v^{2}-5 v}{5(v-2)}$, and

(3) $P_{0}(h)$ is monotone in $\left(h_{u}, h_{v}\right)$ if $\frac{3 v^{2}-5 v}{5(v-2)} \leq u<v$.

Proof. The conclusions (1) and (2) of Theorem 4.2 can be verified by similar methods used in the proof of conclusions (i) and (iii) in Theorem 4.1, respectively.

Using the same notation $\mu(h), \nu(h), s(h), r(h), U(h)$ used in the proof of Theorem 4.1 to represent the corresponding variables, we find that

$$
\frac{d}{d h} s\left(h_{u}\right)=0 \text { as } u=\frac{1}{3}\left(v+1-\sqrt{v^{2}+v+1}\right) .
$$

Thus, as $0<u \leq \frac{1}{3}\left(v+1-\sqrt{v^{2}+v+1}\right)$, similar arguments in the proof of conclusion (i) of Theorem 4.1 can be applied to obtain conclusion (1) of Theorem 4.2 
Also, if $\frac{1}{3}\left(v+1-\sqrt{v^{2}+v+1}\right)<u<\frac{3 v^{2}-5 v}{5(v-2)}$, then following the idea used in the proof of conclusion (iii) of Theorem 4.1, we calculate the derivatives of $P(h)$ at the endpoints of $\left(h_{u}, h_{0}\right)$, and obtain that

$$
\begin{aligned}
& P_{0}^{\prime}\left(h_{u}\right)=\lim _{h \rightarrow h_{u}+} P_{0}^{\prime}(h)=-\frac{3 u^{2}-2 u v-2 u+v}{3 \pi u^{2}(u-v)^{2}(u-1)^{2}}>0 \\
& P_{0}^{\prime}\left(h_{0}\right)=\lim _{h \rightarrow h_{0}-} P_{0}^{\prime}(h)=-\infty<0
\end{aligned}
$$

if $\frac{1}{3}\left(v+1-\sqrt{v^{2}+v+1}\right)<u<\frac{3 v^{2}-5 v}{5(v-2)}$.

Hence, $P(h)$ is not monotone in the interval $\left(h_{u}, h_{0}\right)$ as $\frac{1}{3}\left(v+1-\sqrt{v^{2}+v+1}\right)<$ $u<\frac{3 v^{2}-5 v}{5(v-2)}$, which leads to conclusion $(2)$.

In the following, we will focus on the verification of conclusion (3). We would like to show that $U^{\prime}(h)>0$ in the interval $\left(h_{u}, h_{v}\right)$ if $\frac{3 v^{2}-5 v}{5(v-2)} \leq u<v$. We first claim that $U^{\prime}\left(h_{u}\right)>0$.

Since $\Psi_{0}(\nu(h))=h$, we have that

$$
\begin{aligned}
& \sqrt{h-h_{u}} \\
& =(\nu(h)-u) \sqrt{\frac{u(1-u)(v-u)}{2}+\frac{3 u^{2}-2(v+1) u+v}{3}(\nu(h)-u)+O\left((\nu(h)-u)^{2}\right)} .
\end{aligned}
$$

When $0<h-h_{u} \ll 1$, a straightforward calculation yields the inverse transformation

$$
\begin{aligned}
\nu(h)-u= & \sqrt{\frac{2}{u(1-u)(v-u)} \sqrt{h-h_{u}}} \\
& -\frac{2\left(3 u^{2}-2(v+1) u+v\right)}{3 u^{2}(1-u)^{2}(v-u)^{2}}\left(h-h_{u}\right)+O\left(\left(h-h_{u}\right)^{\frac{3}{2}}\right) .
\end{aligned}
$$

Similarly, because $\Psi_{0}(\mu(h))=h$, we have that

$$
\begin{aligned}
\mu(h)-u= & -\sqrt{\frac{2}{u(1-u)(v-u)}} \sqrt{h-h_{u}} \\
& -\frac{2\left(3 u^{2}-2 u v+v-2 u\right)}{3 u^{2}(1-u)^{2}(v-u)^{2}}\left(h-h_{u}\right)+O\left(\left(h-h_{u}\right)^{\frac{3}{2}}\right) .
\end{aligned}
$$

So

$$
\nu(h)+\mu(h)=2 u-\frac{4\left(3 u^{2}-2(v+1) u+v\right)}{3 u^{2}(1-u)^{2}(v-u)^{2}}\left(h-h_{u}\right)+O\left(\left(h-h_{u}\right)^{\frac{3}{2}}\right) .
$$

Notice that $3 u^{2}-2(v+1) u+v=0$ has two roots, $\frac{1}{3}\left(v+1-\sqrt{v^{2}+v+1}\right)$ and $\frac{1}{3}\left(v+1+\sqrt{v^{2}+v+1}\right)$; hence, $\frac{1}{3}\left(v+1-\sqrt{v^{2}+v+1}\right)<\frac{3 v^{2}-5 v}{5(v-2)}$ and $v<$ $\frac{1}{3}\left(v+1+\sqrt{v^{2}+v+1}\right)$. 
Therefore, when $\frac{3 v^{2}-5 v}{5(v-2)} \leq u<v, 3 u^{2}-2(v+1) u+v<0$, which implies that

$$
U^{\prime}\left(h_{u}\right)=\lim _{h \rightarrow h_{u}^{+}}\left(\mu^{\prime}(h)+\nu^{\prime}(h)\right)=-\frac{4\left(3 u^{2}-2(v+1) u+v\right)}{3 u^{2}(1-u)^{2}(v-u)^{2}}>0 .
$$
have

Next we claim that $U^{\prime}\left(h_{v}\right)>0$. Note that $\Psi_{0}(\nu(h))=h$ and $\Psi_{0}(\mu(h))=h$. We

$$
\begin{aligned}
U^{\prime}\left(h_{v}\right)= & \lim _{h \rightarrow h_{v}^{-}}\left(\mu^{\prime}(h)+\nu^{\prime}(h)\right) \\
= & \lim _{h \rightarrow h_{v}^{-}}\left(\frac{1}{\mu(h)(\mu(h)-u)(\mu(h)-v)(\mu(h)-1)}\right. \\
& \left.\quad+\frac{1}{\nu(h)(\nu(h)-u)(\nu(h)-v)(\nu(h)-1)}\right) \\
= & +\infty,
\end{aligned}
$$

since when $h \rightarrow h_{v}^{-}, \nu(h) \rightarrow v^{-}$and $\mu(h) \rightarrow v_{0}{ }^{+}$, where $v_{0}$ is the $x$-coordinate of the cross point between the positive $x$-axis and the homoclinic loop at the saddle $(v, 0)$, and $0<v_{0}<u$.

Hence, $U^{\prime}(h)>0$ at the endpoints of the interval $\left(h_{u}, h_{v}\right)$ if $\frac{3 v^{2}-5 v}{5(v-2)} \leq u<v$.

We now discuss the relative position of $U\left(h_{u}\right)$ and $U\left(h_{v}\right)$.

If $v \geq 2 u$, then $U\left(h_{v}\right)=v+\mu\left(h_{v}\right)>U\left(h_{u}\right)=2 u$, where $\mu\left(h_{v}\right)$ is the coordinate of the cross point of the homoclinic loop $\Gamma_{0}\left(h_{v}\right)$ and the $x$-axis, and $0<\mu\left(h_{v}\right)<u$.

If $v<2 u$, then $0<U\left(h_{v}\right)$ and $2 u-v<u$. A straightforward calculation shows that

$$
\Psi_{0}\left(U\left(h_{v}\right)\right)-\Psi(2 u-v)=\frac{(v-u)^{3}\left(3 v^{2}+(5-16 u) v+2 u(9 u-5)\right)}{15}
$$

and $3 v^{2}+(5-16 u) v+2 u(9 u-5)<0$ if $v=u$ or $v=2 u$.

Hence, if $u<v<2 u$, then the quadratic polynomial

$$
3 v^{2}+(5-16 u) v+2 u(9 u-5)<0,
$$

which implies that $\Psi_{0}\left(U\left(h_{v}\right)\right)<\Psi(2 u-v)$.

Notice that $\Psi_{0}(x)$ is decreasing in $(0, u)$. Thus, $U\left(h_{v}\right)>2 u-v$ and

$$
U\left(h_{v}\right)=v+\mu\left(h_{v}\right)>U\left(h_{u}\right)=2 u .
$$

Now we are in a position to prove that $U^{\prime}(h)>0$ for $h \in\left(h_{u}, h_{v}\right)$.

By contradiction, we assume that $U^{\prime}(h)$ has zeros in $\left(h_{u}, h_{v}\right)$. Then $U^{\prime}(h)$ has at least two zeros since $U^{\prime}\left(h_{u}\right)>0$ and $U^{\prime}\left(h_{v}\right)>0$. As shown in Figure 3. since $U\left(h_{v}\right)>U\left(h_{u}\right)$, there exists a $u_{0}$ such that $U(h)=u_{0}$ has at least three distinct roots $\hat{h}<h^{*}<\breve{h}$.

Consider the new function

$$
\tau(h) \triangleq \mu(h) \nu(h) .
$$

It is easy to obtain that $\tau(h)=\frac{U^{2}(h)}{4}-r^{2}(h)$ by the definitions of $\mu(h), \nu(h), U(h)$ and $r(h)$.

Since $U(\hat{h})=U\left(h^{*}\right)=u(\breve{h})=u_{0}$ and $0<r(\hat{h})<r\left(h^{*}\right)<r(\breve{h}), \tau(\hat{h}), \tau\left(h^{*}\right)$ and $\tau(\breve{h})$ are different from each other. 


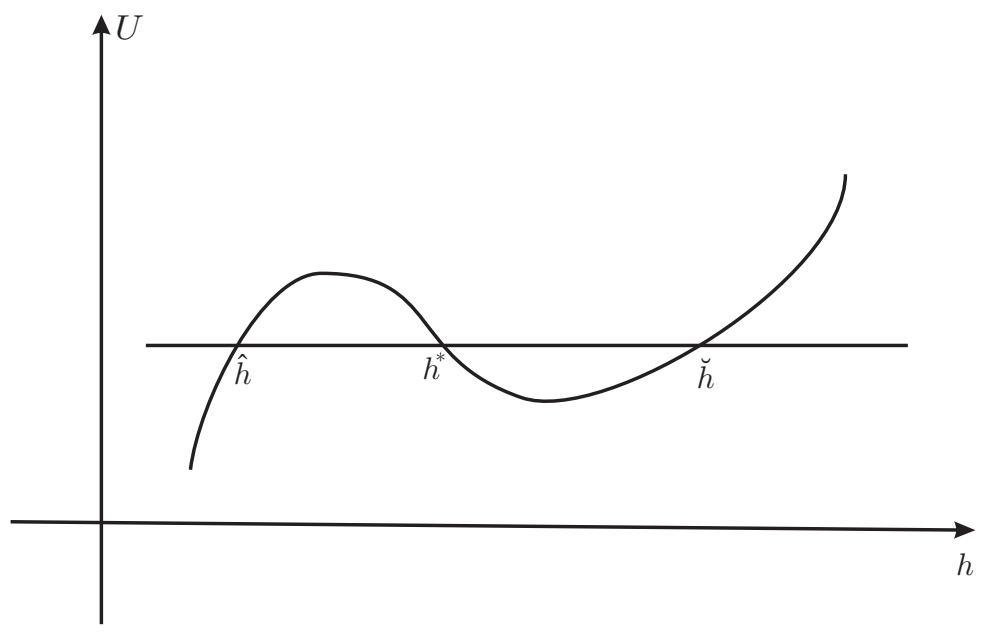

Figure 3. The hypothetical figure of $U(h)$.

Substituting $\mu(h)+\nu(h)=U(h)$ and $\mu(h) v(h)=\tau(h)$ to $\Psi_{0}(\mu(h))=\Psi_{0}(\nu(h))$, we obtain

$$
\begin{aligned}
12 \tau^{2}(h)+\tau(h)(-20 u+30 U(h) & \left.-36 U^{2}(h)-20 u v-20 v+30 u U(h)+30 v U(h)\right) \\
+ & U(h)\left(12 U^{3}(h)-15 u U^{2}(h)-15 U^{2}(h)-15 v U^{2}(h)\right. \\
+ & 20 v U(h)+20 u U(h)+20 u v U(h)-30 u v)=0 .
\end{aligned}
$$

Let $U(h)=u_{0}$. Then $r(\hat{h}), r\left(h^{*}\right)$ and $r(\breve{h})$ must satisfy the above equation. However, the above equation has at most two zeros. This leads to a contradiction. Thus, $U^{\prime}(h)$ has the fixed sign in $\left(h_{u}, h_{v}\right)$ and is positive. By Theorem 2.1, the function $P_{0}(h)$ is monotone in $\left(h_{u}, h_{v}\right)$ if $\frac{3 v^{2}-5 v}{5(v-2)} \leq u<v$. We complete the proof.

From Theorem 4.2, we know that there is a curve

$$
C=\left\{(u, v): u=\frac{1}{3}\left(v+1-\sqrt{v^{2}+v+1}\right), 0 \leq v \leq 1\right\}
$$

between $L_{v}$ and $\gamma$ in the bifurcation diagram (see Figure 2) of system (4.9), which is the bifurcation curve of the number of isolated zero of the Abelian integral

$$
I(h)=\beta_{0} \oint_{\Gamma_{0}(h)} y d x+\beta_{1} \oint_{\Gamma_{0}(h)} x y d x=\beta_{0} I_{0}(h)+\beta_{1} I_{1}(h),
$$

where $\beta_{0}$ and $\beta_{1}$ are any real numbers.

Based on Theorems 4.1 and 4.2 , we obtain the following corollary on the number of isolated zeros of $I(h)$.

Corollary 4.1. For any real $\beta_{0}$ and $\beta_{1}$, the Abelian integral $I(h)$ has at most one isolated zero if one of the following conditions holds:

(i) Parameters $u$ and $v$ are in the region between $L_{v}$ and $C$, that is,

$$
(u, v) \in\left\{(u, v): 0 \leq u \leq \frac{1}{3}\left(v+1-\sqrt{v^{2}+v+1}\right), 0 \leq v \leq 1\right\}
$$


(ii) $(u, v)=\left(\frac{2}{5}, 1\right)$ and $(u, v)=(1,1)$;

(iii) $u=\bar{v}$ and $(\operatorname{Re}(u)-2)^{2}+(\operatorname{Im} v)^{2} \geq 1$.

Also, there exist some real values of $\beta_{0}$ and $\beta_{1}$ such that the Abelian integral $I(h)$ has at least two isolated zeros if one of the following conditions holds:

(a) Parameters $u$ and $v$ are in the region between $C$ and $\gamma$, that is,

$$
(u, v) \in\left\{(u, v): \frac{1}{3}\left(v+1-\sqrt{v^{2}+v+1}\right)<u<\frac{3 v^{2}-5 v}{5(v-2)}, 0<v \leq 1\right\} ;
$$

(b) $u=\bar{v}$ and $(\operatorname{Re}(u)-2)^{2}+(\operatorname{Im} v)^{2}<1$.

Remark 4.1. Note that if $u$ and $v$ are in the region between $\gamma$ and $L_{u v}$, that is,

$$
(u, v) \in\left\{(u, v): \frac{3 v^{2}-5 v}{5(v-2)}<u \leq v, 0<v<1\right\},
$$

there exist big ovals $\Gamma_{0}(h)$ which surround two equilibria or three equilibria. In these cases, the monotonicity of $P_{0}(h)$ are more complicated and our criterion cannot be applied to deal with them. If we only consider two continuous families $J_{1}$ and $J_{u}$ of ovals, each of them surrounds only one equilibrium as $u$ and $v$ are in the region between $\gamma$ and $L_{u v}$, then the Abelian integral $I(h)$ has at most one isolated zero in the interval $J_{1} \cup J_{u}$ for any real $\beta_{0}$ and $\beta_{1}$.

Last let us discuss the main results in [10] and our results. Gavrilov and Iliev in [10] studied the monotonicity of the ratio of the derivatives of $I_{1}(h)$ and $I_{0}(h)$, that is, the monotonicity of $\frac{I_{1}^{\prime}(h)}{I_{0}^{\prime}(h)}$. By the Riemann bilinear relations on differentials of the first kind together with the fact that a Jacobian variety with its polarization cannot be a direct product of principally polarized Abelian varieties, they obtained the main result: exceptional families of ovals $\Gamma_{0}(h)$ of system (4.8) exist such that $\frac{I_{1}^{\prime}(h)}{I_{0}^{\prime}(h)}$ is monotone. In the real case $(0<u<v<1)$, the continuous family of ovals $\Gamma_{0}(h)$ surrounding the center $(1,0)$ is exceptional, and in the complex case $(u=\bar{v})$, the continuous family of ovals $\Gamma_{0}(h)$ surrounding the center $(1,0)$ is exceptional if and only if $(\operatorname{Re}(u)-2)^{2}+(\operatorname{Im} v)^{2} \geq 1$.

From conclusions (i) and (ii) in Theorem 4.1, one can see that for exceptional families in [10], $\frac{I_{1}(h)}{I_{0}(h)}$ is monotone too.

Gavrilov and Iliev in [10] also stated that in the real case $(0<u<v<1)$, the continuous family of ovals $\Gamma_{0}(h)$ surrounding the center $(u, 0)$ is not exceptional, and in the complex case $(u=\bar{v})$, the continuous family of ovals $\Gamma_{0}(h)$ surrounding the center $(1,0)$ is not exceptional if $(\operatorname{Re}(u)-2)^{2}+(\operatorname{Im} v)^{2}<1$. For the nonexceptional family, they gave some conjectures on the Chebyshev with accuracy at most one or two for $\beta_{0} I_{0}^{\prime}(h)+\beta_{1} I_{1}^{\prime}(h)$.

From conclusion (iii) in Theorem 4.1 and conclusion (2) in Theorem 4.2, we can see that for the non-exceptional family in the complex case and the real case $\frac{1}{3}\left(v+1-\sqrt{v^{2}+v+1}\right)<u<\frac{3 v^{2}-5 v}{5(v-2)}, \beta_{0} I_{0}(h)+\beta_{1} I_{1}(h)$ is Chebyshev with accuracy of at least one. However, for the non-exceptional family in the other real cases, that is, either $0<u \leq \frac{1}{3}\left(v+1-\sqrt{v^{2}+v+1}\right)$ or $\frac{3 v^{2}-5 v}{5(v-2)} \leq u<v, \beta_{0} I_{0}(h)+\beta_{1} I_{1}(h)$ is Chebyshev by conclusions (1) and (3) in Theorem 4.2. Therefore, the classification of the exceptional family and non-exceptional family on the continuous family of ovals surrounding a center is not exact classification for the corresponding Abelian integrals to have Chebyshev property. 


\section{ACKNOWLEDGEMENTS}

The authors express their gratitude to the anonymous referee and editor for their valuable comments and suggestions.

\section{REFERENCES}

1. V. I. Arnold, Geometrical Methods in the Theory of Ordinary Differential Equations, SpringerVerlag, 1983. MR695786(84d:58023)

2. V. I. Arnold, Ten Problems, in: Theory of singularities and its applications, Advance in Soviet Mathematics, 1(1990), 1-8. MR1089668 (91k:58001)

3. A. Atabaigi and H.Z. Zangeneh, Bifurcation of limit cycles in small perturbations of a class of hyperelliptic Hamiltonian systems of degree 5 with a cusp, J. Appl. Anal. Comp., 1 (2011), 299-313.

4. G. Binyamini, D. Novikov and S. Yakovenko, On the number of zeros of Abelian integrals, Invent. Math., 181 (2010), 227-289. MR2657426 (2011m:34089)

5. R. I. Bogdanov, Versal deformation of a singularity of a vector field on the plane in case of zero eigenvalues, Selecta Math. Soviet., 1 (1981), 389-421.

6. F. Dumortier and C. Li, Perturbations from an elliptic Hamiltonian of degree four I, Saddle loop and two saddle cycle, J. Diff. Eqns., 176 (2001), 114-157. MR1861185 (2002h:34061)

7. F. Dumortier and C. Li, Perturbations from an elliptic Hamiltonian of degree four II, Cuspidal loop, J. Diff. Eqns., 175 (2001), 209-243. MR1855970 (2002h:34060)

8. F. Dumortier and C. Li, Perturbations from an elliptic Hamiltonian of degree four III, Global centre, J. Diff. Eqns., 188 (2001), 473-511. MR1954291 (2003j:34046a)

9. F. Dumortier and C. Li, Perturbation from an elliptic Hamiltonian of degree four IV. Figure eight-loop, J. Diff. Eqns., 188 (2003), 512-554. MR.1954292 (2003j:34046b)

10. L. Gavrilov and I.D. Iliev, Completle hyperelliptic integrals of the first kind and their nonoscillation, Trans. Amer. Math. Soc., 356 (2003), 1185-1207. MR2021617 (2004i:34077)

11. M. Grau, F. Mañosas and J. Villadelprat, A Chebyshev criterion for Abelian integrals, Trans. Amer. Math. Soc., 363 (2011), 109-129. MR2719674 (2011j:34096)

12. R. Kazemi, H.Z. Zangeneh and A. Atabaigi, On the number of limit cycles in small perturbations of a class of hyperelliptic Hamiltonian systems, Nonlinear Analysis: Theory, Methods \& Appl., 76 (2012), 574-587.

13. C. Li, Abelian Integrals and Limit Cycles, Qual. Theory Dyn. Syst., 11 (2012), 111-128. MR.2902727

14. C. Li and Z. Zhang, A criterion for determining the monotonocity of the ratio of two Abelian integrals, J. Diff. Eqns., 124 (1996), 407-424. MR.1370149 (96i:34057)

15. J. Li, Two results on Lienard equations, Ph.D. Thesis, Peking University, 1998.

16. C. Liu, Estimate of the Number of Zeros of Abelian Integral for Elliptic Hamiltonian with Figure Eight-Loop, Nonlinearity, 16 (2003), 1151-1163. MR.1975800 (2003m:34077)

17. D. Novikov and S. Yakovenko, Tangential Hilbert Problem for perturbations of Hyperelliptic Hamiltonian Systems, Electron. Announc. Amer. Math. Soc., 5 (1999), 55-65. MR 1679454 (2000a:34065)

18. G. S. Petrov, Elliptic integrals and their nonoscillation (Russian), Funct. Anal. Appl., 20 (1986), no. 1, 46-49. MR831048 (87f:58031)

19. G. S. Petrov, Complex zeros of an elliptic integral, Func. Anal. Appl., 23 (1989), 88-89. MR1011373 (90m:33002)

20. G. S. Petrov, Nonoscillation of elliptic integrals, Func. Anal. Appl., 24 (1990), 205-210. MR.1082030 (92c:33036)

21. G. S. Petrov, On the nonoscillation of elliptic integrals, Funct. Anal. Appl., 31 (1997), 262265. MR1608896 (99a:34087)

22. R. Roussarie, On the number of limit cycles which appear by separatrix loop of planar vector fields, Bol. Soc. Bra. Math., 17 (1986), 67-101. MR.901596 (88i:34061)

23. D. Shang and T. Zhang, Bifurcations of a cubic system perturbed by degree five, Acta Math. Sci. Ser. B Engl. Ed., 29 (2009), 11-24. MR2494080 (2010b:34064)

24. J. Wang and D. Xiao, On the number of limit cycles in small perturbations of a class of hyperelliptic Hamiltonian systems with one nilpotent saddle, J. Diff. Equa., 250 (2011), 22272243. MR2763571(2012c:34087) 
25. T. Zhang, M. Tadé and Y. Tian, On the zeros of the Abelian integrals for a class of Liénard systems, Phys. Lett. A, 358 (2006), 262-274. MR2253504(2007g:34063)

26. T. Zhang, Y. Tian and M. Tadé, On the number of zeros of the abelian integrals for a class of perturbed Liénard systems, Internat. J. Bifur. Chaos Appl. Sci. Engrg., 17 (2007), 3281-3287. MR2372300 (2008k:34130)

27. Y. Zhao and Zhifen Zhang, Linear estimate of the number of zeros of abelian integrals for a kind of quartic hamiltonians, J. Diff. Equa., 155 (1999), 73-88. MR.1693214 (2000g:34052)

School of Mathematics, Soochow University, Suzhou 215006, People's Republic of China

E-mail address: liucj@suda.edu.cn

Department of Mathematics, Shanghai Jiao Tong University, Shanghai 200240, PeoPle's Republic OF ChinA

E-mail address: xiaodm@sjtu.edu.cn 